Recepción: 10 / 03 / 2018

Aceptación: 14 / 05 / 2018

Publicación: 05 / 06 / 2018
Ciencias económicas y empresariales

Artículo de Investigación

\title{
Emprendimiento y Gerencia Integral en los nuevos tiempos
}

\section{Entrepreneurship and Integral Management in the new times}

\author{
Emprestimiento y Gerencia Integral en los nuevos tiempos
}

\author{
Cecivel A. Velasco-Guachamin ${ }^{\mathrm{I}}$ \\ cavelasco9@espe.edu.ec \\ Cristian S. Viteri-Arias II \\ csviteri1@espe.edu.ec \\ Nilton A. Guayta-Toapanta ${ }^{\text {III }}$ \\ alexeins_69@hotmail.com
}

Correspondencia: cavelasco9@espe.edu.ec

\footnotetext{
I Ingeniera en Contabilidad y Auditoría CPA, Docente de la ESPE, Latacunga, Ecuador.

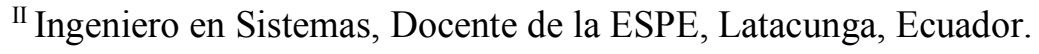

${ }^{\mathrm{III}}$ Ingeniero Comercial, ESPE, Latacunga, Ecuador.
} 


\title{
Resumen
}

El estudio con respecto a las nuevas manifestaciones empresariales y las nuevas formas de organizaciones, es de sumo de interés, toda vez que reportan hallazgos que pueden utilizarse para mejorar los ingresos y aumentar la competitividad de las empresas del país. Por tal razón, este estudio se propuso conocer si los nuevos emprendimientos ecuatorianos contemplan o no la Gerencia Integral como práctica para dirigir sus empresas. El estudio fue no experimental y descriptivo. Se realizó una indagación en cuanto al perfil del gerente integral a partir de los aspectos teóricos y luego una indagación empírica en 48 emprendimientos del país. Para recolectar la información se aplicaron dos cuestionarios que se analizaron mediante distribución de frecuencias. Entre los resultados obtenidos se destaca la presencia de varios rasgos del gerente integral en los emprendimientos estudiados, lo cual incide directamente en el éxito de las empresas y en sus niveles de competitividad en el mercado.

Palabras clave: Manifestaciones empresariales; ingresos; competitividad; gerencia integral.

\begin{abstract}
The study of new business manifestations and new forms of organizations is of interest, since they report findings that can be used to improve revenues and increase the competitiveness of companies in the country. For this reason, this study set out to know if the new Ecuadorian enterprises contemplate or not the Integral Management as practice to direct their companies. The study was non-experimental and descriptive. An inquiry was made as to the profile of the integral manager based on the theoretical aspects and then an empirical inquiry into 48 ventures of the country. To collect the information, two questionnaires were applied and analyzed by frequency distribution. Among the results obtained, we highlight the presence of several features of the integral manager in the projects studied, which directly affects the success of companies and their levels of competitiveness in the market.
\end{abstract}

Keywords: Business manifestations; income; competitiveness; integral management.

\section{Resumo}

O projeto consiste nas novas manifestações empresariais e as novas formas de organização, são de sumo de interêses, toda vez que os relatórios são aplicáveis que aumentam a taxa de 
crescimento e aumentam a competitividade das empresas do país. Por tal razao, este estudio proprio conocer e los nuevos emprendimientos ecuatorianos contemplan on no la Gerencias Integral como práctica para o transporte de empresas. El estudio fue não experimental y descritivo. Você está realizando uma indagação em relação ao perfil da gerente integral a partir das funções teóricas e jurídicas da empresa empírica em 48 empreedimientos del país. Para obter informações sobre a aplicação dos custos que se analizam mediante distribuição de frecuencias. Entre os resultados obtidos destaca-se a presença de varios gestores da empresa integral nos empreendimentos estudados, e incide directamente no éxito das empresas e em todos os eventos de competitividade no mercado.

Palavras chave: Manifestaciones empresariaises; ingresos; competitividad; integral de gerencia.

\section{Introducción}

Entre los distintos campos que se despliegan hoy día a partir de la administración y la gerencia, aparece uno que luce de relevancia dadas las actuales demandas altamente competitivas de un mundo complejizado por el mercado, y la necesidad de crear otros modelos y modos de crecimiento. Se trata de la gerencia integral, propuesta por Sallenave en 2002, la cual tiene que ver con el estudio de la Acción Empresarial y entender, desde un enfoque no parcelado, los elementos que inciden en el éxito o fracaso de la empresa frente a sus competidores.

Después de un largo y continuado intento de promover nuevas ideas cuyo fin ha sido "reducir la complejidad del fenómeno empresarial a un solo componente que, supuestamente, puede explicarlo todo" (Sallenave, 2002), la gerencia integral luce como un enfoque holístico desde el cual dicha Acción Empresarial se pueda estudiar más allá de la visión fragmentaria del conocimiento organizacional y administrativo.

Como quiera que se aprecie, todos estos esfuerzos se vinculan al fin último que persigue toda organización en el mundo de los negocios, el cual es incrementar su productividad, obtener un lugar competitivo en su ramo, y, con ello, garantizar un óptimo flujo de ganancias con menor costo. El logro de este propósito, está vinculado con una serie de factores que le dan vida a la organización, y estos factores son los que han motivado las diversas ramas de estudio dentro de las ciencias de la administración, todas las cuales se integran al ser estudiadas bajo la perspectiva de la gerencia integral, sin escisiones entre ellas. 
Este enfoque está también enlazado con otro no tan reciente pero aún vigente, que es el de la gerencia y planificación estratégica, que supone la orientación para la toma de decisiones de modo eficiente y eficaz, dando el uso más óptimo posible a los diversos recursos con que cuenta la empresa u organización. Bajo esta perspectiva, vale afirmar que la gestión estratégica consiste en un sistema integrado e interrelacionado, que inicia con el análisis estratégico del entorno de la organización en sus diferentes dimensiones y niveles; continuando con la planeación estratégica; después, se determinan y evalúan las alternativas estratégicas disponibles y se establece el alcance de la estrategia; se realiza la formulación estratégica, principalmente a través de proyectos, planes de acción y presupuestos estratégicos; posteriormente, se procede a la implementación que requiere entre otros aspectos un alto grado de liderazgo, y finalmente, se adelanta el control estratégico y de gestión. (García, Bolívar, \& Roa, 2011)

Esta articulación entre gerencia integral y planificación o planeación estratégica, también se enrumba con especial énfasis hacia el perfil del gerente, cuyas características contemplan un conjunto de potencialidades, capacidades, habilidades y conocimientos que van desde aspectos financieros hasta un perfil humano integral y asertivo. Desde este punto de vista, una de las premisas fundamentales para el éxito organizacional radica en las cualidades esenciales que debe poseer el dirigente de empresa, quien, en primer lugar, debe asumir la planeación estratégica como un proyecto de vida, para luego desarrollar la planeación estratégica como un proyecto organizacional contextualizado y pertinente. (Román, Arbeláez, \& Patiño, 2012)

Para estudiar estos aspectos, este trabajo también aborda una tendencia emergente en el mundo empresarial llamado emprendimiento, cuyo desarrollo ha venido madurando progresivamente, para convertirse en una nueva rama de la administración dentro de la cual se promueve la creatividad, el empuje y la iniciativa hacia nuevos proyectos empresariales públicos o privados; esto en tanto el mismo ha emergido en la realidad misma del mercado, y requiere nuevos dispositivos para su estudio y sistematización.

Adicionalmente, desde el punto de vista teórico y académico, en los estudios sobre el emprendimiento se ha venido hablando también de nuevas formas de organización, otras modalidades de gerencia, frescas y renovadas, más flexibles y adecuadas a la veloz dinámica social actual, con nuevos lenguajes y códigos, nuevas relaciones y nuevas soluciones. 
En este punto se puede mencionar lo señalado por Lederman, Messsina, Pienknagura y Rigolini (2014), cuando afirman que "el emprendimiento comprende la entrada de empresas en mercados nuevos o ya establecidos (tanto nacionales como extranjeros), la introducción de productos nuevos en el mercado y los avances organizativos que permiten a las empresas mejorar la calidad o el precio de los productos o adoptar maneras de producir más eficientes". (p. 3)

Así como en el caso de la Gerencia Integral y de la planificación estratégica, los rasgos del líder emprendedor, son sumamente importantes, ya que de ellos derivará el éxito o no del emprendimiento y, por ende, su productividad y competitividad en el área en la cual se inserta. En consecuencia, cabe afirmar que los emprendedores de éxito son individuos que transforman ideas en iniciativas rentables. A menudo, esta transformación requiere talentos especiales, como la capacidad de innovar, introducir nuevos productos y explorar otros mercados. Se trata de un proceso que también precisa la habilidad de dirigir a otras personas, priorizar las tareas para aumentar la eficiencia productiva y dar a los recursos disponibles el mejor uso posible. (Lederman, Julián, Pienknagura, \& Rigolini, 2014)

Vale la pena considerar que Ecuador en los últimos años ha tenido un incremento significativo de los emprendimientos y, tal como aparece reseñado por Araque (2015), tomando como referencia el Censo Nacional Económico de 2010:

Alrededor del 99\% de establecimientos empresariales, nacieron bajo la categoría de actividad microempresarial, registrándose un número promedio de dos trabajadores contratados y una inversión financiera media de USD 7.289 -incluye capital fijo y capital de trabajo- por cada microempresa que inició operaciones desde el 2010.

Este creciente fenómeno está orientado hacia áreas diversas, aunque con especial énfasis en dos macro sectores: "comercio (53\%), y servicio (39\%), quedando en alrededor del 8\% para iniciativas emprendedoras ubicadas como actividades manufactureras" (Ob. Cit.). Con base en el mencionado reporte de Araque (2015), la inversión se ha dirigido hacia rubros tales como: comercio, reparación de vehículos, alojamiento y comida, comunicación e información y manufacturas.

Visto este contexto, conviene mencionar que el propósito fundamental planteado para este estudio, fue conocer si los nuevos emprendimientos ecuatorianos contemplan o no la Gerencia 
Integral (conscientemente o no) como práctica para dirigir sus empresas. Del mismo modo, se planteó una indagación en torno a las características de los emprendedores en cuanto al perfil del gerente integral. Para ello, era fundamental iniciar por determinar cuáles son las características de la gerencia y del gerente integral según los fundamentos teóricos necesarios.

Hay que advertir que este tipo de investigaciones revisten especial interés, ya que contienen elementos actualizados para comprender el comportamiento organizacional en los nuevos tiempos, las lógicas que imperan en estas nuevas iniciativas y sus dinámicas, y aportan elementos que permiten mejorar la competitividad de las empresas y organizaciones, todo lo cual redunda en el desarrollo comercial y productivo del país, al generar un mercado más competitivo y con mayor circulación de recursos.

\section{Metodología}

Para dar cumplimiento a los propósitos propuestos para el estudio, éste se considera, primeramente, no experimental, el cual, según Hernández, Fernández, \& Baptista (2010), “se realizan sin la manipulación deliberada de variables y en los que sólo se observan los fenómenos en su ambiente natural para después analizarlos" (p.149). Esto es así en tanto que se observa una situación que ya existe sin manipulaciones intencionales por parte del investigador, por ende, no hay control sobre las variables que se presentan en el objeto estudiado.

Seguido, tomando en consideración lo novedoso de la temática, el trabajo se enmarca dentro de lo que es un diseño transaccional descriptivo, el cual, según los mismos Hernández, Fernández, \& Baptista (2010), tiene como objetivo indagar la incidencia de las modalidades o niveles de una o más variables en una población. El procedimiento consiste en ubicar en una o diversas variables a un grupo de personas u otros seres vivos, objetos, situaciones, contextos, fenómenos, comunidades; y así proporcionar su descripción. (p. 153)

Con respecto a la descripción, Monje (2011), sostiene que se procede, con base en la información obtenida, a ordenar los rasgos, atributos o características de la realidad observada de acuerdo con el problema (...) la descripción se ocupa fundamentalmente de la información sobre cantidad, ubicación, capacidad, tipo y situación general del problema (...) lleva al investigador a presentar los hechos y eventos que caracterizan la realidad observada tal como ocurren. (p. 95) 
Para ejecutar el trabajo, se utilizó una muestra no probabilística, seleccionar aleatoriamente varios emprendimientos de diversos ramos para observarlos y estudiar a sus líderes emprendedores de acuerdo a los presupuestos de la gerencia integral. La muestra de los emprendimientos estudiados quedó compuesta de la siguiente manera:

Tabla 1. Composición de la Muestra

\begin{tabular}{|l|c|}
\hline \multicolumn{1}{|c|}{ DESCRIPCIÓN } & CANTIDAD \\
\hline Comercios mayoristas & 8 \\
\hline Comercios al detal & 5 \\
\hline Talleres de vehículos & 4 \\
\hline Talleres de electrodomésticos & 3 \\
\hline Talleres de equipos celulares y computadoras & 6 \\
\hline Servicios de comida & 6 \\
\hline Servicios de comunicación e información & 8 \\
\hline Servicios de estética & 8 \\
\hline TOTAL & 48 \\
\hline
\end{tabular}

La información se obtuvo mediante la aplicación de dos (02) cuestionarios, uno dirigido a los líderes emprendedores y otro dirigido a sus empleados. Con ellos se buscó determinar cuáles rasgos derivados de la gerencia integral, de la planificación estratégica, estaban presentes en estos emprendimientos y en sus líderes emprendedores.

Adicionalmente, se hizo uso de la investigación documental, para extraer todos los aspectos que formarían parte de los instrumentos diseñados para la indagación, y se analizaron mediante una distribución de frecuencias, entendida como "un conjunto de puntuaciones presentadas en una tabla de manera ordenada, según características definidas por el investigador” (Bernal, 2010). Las cifras arrojadas por esta distribución se sometieron a un análisis por parte del autor.

\section{Resultados}

En primer lugar, se procedió a determinar los aspectos característicos de la gerencia integral y del líder emprendedor a partir de la fundamentación de dichas variables teóricas. Tales hallazgos se muestran a continuación: 


\section{Sobre los rasgos del líder emprendedor desde la Gerencia Integral}

Se puede saber si en una empresa existe o no una gerencia integral indistintamente del tamaño de la organización. Para ello, se tomarán como elementos característicos los establecidos por Sallenave (2002), cuando sostiene que:

La Gerencia Integral es el arte de reunir todas las facetas del manejo de una organización en busca de una mayor competitividad:

- La estrategia: Para saber a dónde vamos y como lograrlo.

- La organización: Para llevar a cabo la estrategia eficientemente.

- La cultura: Para dinamizar la organización y animar a su gente (p. 3)

Desde esta particular perspectiva, este autor señala que le gerente integral reúne características vinculadas con estas tres facetas, a saber: estratega, organizador y líder. El rasgo de estratega, forja el porvenir de su empresa (...) adquiere una visión de lo que podría ser la empresa del mañana. De esta visión deriva la misión de la empresa, es decir, la articulación de su razón de ser y de sus objetivos esenciales. La misión delimita el campo de acción de la planificación estratégica porque todas las estrategias que el gerente formule deben caber dentro de la misión y deben ayudar a que ésta se cumpla. La última función del gerente-estratega es la implementación y el control de la estrategia. (Ob. Cit., p.24).

Por su parte, el carácter organizador implica "darle a un grupo de personas los medios para realizar una tarea" (Ob. Cit,p. 25); define las tareas y las líneas de autoridad y responsabilidad, debe conocer con claridad la estructura y las relaciones que la hacen funcionar, asimismo, debe conocer suficientemente las características y habilidades del talento humano con que cuenta para cada tarea, así como sus respectivas limitaciones.

Finalmente, la característica de líder hace especial énfasis en el aspecto humano del emprendedor y de quienes componen la empresa, tiene marcado carácter intersubjetivo y lleva implícita la sensibilidad hacia los demás y su entorno. Del mismo modo, este líder de rasgos humanos y sensibles, debe conocer los elementos que inciden en las interrelaciones dentro de la empresa, derivados de la estructura y derivados de la propia subjetividad de los individuos que la componen. 
Para complementar este aspecto en particular, se consideró relevante el trabajo realizado por Moya \& Santana (2016), ya que contiene una pormenorizada sistematización teórica acerca de las diversas definiciones de emprendimiento y el devenir histórico de dichos planteamientos hasta la actualidad, mostrando las tendencias contemporáneas al respecto. Por ende, de estos autores se toman las Competencias para el emprendimiento que se muestran en la siguiente tabla:

Tabla 2. Competencias del Líder Emprendedor

\begin{tabular}{|c|c|}
\hline Competencia & Definición \\
\hline Motivación & $\begin{array}{l}\text { Conjunto de razones por las que las personas se comportan de } \\
\text { la forma en que lo hacen. } \\
\text { El comportamiento motivado es vigoroso, dirigido y sostenido } \\
\text { (...) es el elemento central que conduce lo que la persona } \\
\text { realiza y hacia qué objetivos se dirige. El sujeto se plantea un } \\
\text { objetivo, utiliza los recursos adecuados y mantiene una } \\
\text { determinada conducta, con el propósito de lograr una meta }\end{array}$ \\
\hline Autoeficacia & $\begin{array}{l}\text { Hace referencia al juicio personal del individuo sobre sus } \\
\text { capacidades para enfrentar determinadas situaciones. Así, las } \\
\text { personas tenderían a evitar situaciones de riesgo que } \\
\text { consideran superiores a sus habilidades para afrontarlas, } \\
\text { mientras que se comprometerán con mayor seguridad en las } \\
\text { actividades que se creen capaces de realizar. }\end{array}$ \\
\hline $\begin{array}{l}\text { Reconocimiento } \\
\text { de } \\
\text { oportunidades }\end{array}$ & $\begin{array}{l}\text { La oportunidad es aquella situación en la que los nuevos } \\
\text { productos, servicios, materias primas y métodos organizativos } \\
\text { pueden ser introducidos y vendidos en el mercado a un precio } \\
\text { superior al coste de producirlos. El reconocimiento de la } \\
\text { oportunidad debe ser entendido como un proceso cognitivo a } \\
\text { través del cual los individuos concluyen con la identificación de } \\
\text { una oportunidad. Incluye tres procesos diferenciados: la } \\
\text { percepción (es el reconocimiento por parte de un individuo de } \\
\text { la existencia de necesidades de mercado no satisfechas o de } \\
\text { recursos infrautilizados que pueden ser distribuidos de una } \\
\text { manera más eficiente), el descubrimiento (fase en la que el } \\
\text { individuo analiza la oferta actual en términos de recursos- } \\
\text { producto o servicio-mercado para indagar sobre nuevos ajustes } \\
\text { posibles generados de una forma más eficiente y/o que aporten } \\
\text { un valor añadido al mercado) y la creación del concepto de } \\
\text { negocio (es el reconocimiento de la mejor solución para } \\
\text { satisfacer las necesidades del mercado) }\end{array}$ \\
\hline $\begin{array}{l}\text { Explotación de } \\
\text { oportunidades }\end{array}$ & $\begin{array}{l}\text { Materialización organizativa de la idea emprendedora } \\
\text { identificada y en la que los recursos y capacidades del } \\
\text { emprendimiento creado influyen en su viabilidad técnica y } \\
\text { financiera. Se enfoca en factores como rápida capacidad para } \\
\text { la toma de decisiones y flexibilidad organizacional a través de } \\
\text { los cuales responder a los nuevos mercados y a las } \\
\text { oportunidades tecnológicas }\end{array}$ \\
\hline $\begin{array}{l}\text { Toma de } \\
\text { decisiones bajo } \\
\text { incertidumbre }\end{array}$ & $\begin{array}{l}\text { Se dice que una decisión se toma bajo incertidumbre cuando } \\
\text { no es posible asignar probabilidades a los eventos posibles, es } \\
\text { decir, quien toma la decisión no tiene conocimiento sobre } \\
\text { ningún estado de la naturaleza del resultado o sobre los costos } \\
\text { que le llevará conseguir la información necesaria evitar la } \\
\text { incertidumbre. En estos casos la decisión, además de por } \\
\text { criterios políticos y económicos, se ve orientada por la } \\
\text { orientación psicológica del decisor. }\end{array}$ \\
\hline
\end{tabular}

Fuente: (Moya \& Santana, 2016) 
A partir de estas precisiones teóricas, se elaboraron los instrumentos que permitieron indagar en los emprendimientos seleccionados, las características de la gerencia y de sus líderes, para conocer si estaban presentes o no estos rasgos en los casos estudiados. En función de ello, los instrumentos arrojaron, entre otras, las siguientes informaciones:

- $62 \%$ de la muestra estudiada, cuenta con gerentes estrategas, es decir, sólo este porcentaje conoce, reconoce y pone en práctica los elementos de la gerencia estratégica, tomando en cuenta elementos formales como la visión, misión, planificación estratégicas y control del proceso. Pese a ello, sí realizan monitoreo y control de las diferentes actividades y hay claridad empírica con respecto a los objetivos de la organización.

- $86 \%$ tiene gerentes organizadores, que conocen bien las características de sus empresas y de los recursos materiales y humanos con que cuentan, razón por la cual organizan eficientemente las tareas y las responsabilidades dentro del personal.

- 68\% evidencian los rasgos del líder humano y sensible anteriormente descrito, lo cual nos deja un porcentaje significativo sin este rasgo tan importante.

- $95 \%$ muestra rasgos de motivación y su accionar se encuentra claramente orientado al logro de los objetivos trazados.

- $81 \%$ son autoeficaces, es decir, confía en sus propias habilidades para enfrentar novedades y situaciones potencialmente riesgosas, y confían en sus equipos de trabajo para apoyar las acciones a que haya lugar.

- 73\% reconoce las nuevas oportunidades. De hecho, este rasgo es el que da origen a los diversos emprendimientos, ya que se trata de propuestas en el marco de una demanda alta en el mercado que requiere ser atendida o de situaciones novedosas que generaban competitividad.

- 73\% explota esas oportunidades de negocio, lo cual ratifica el resultado anterior.

- $61 \%$ toma decisiones bajo incertidumbre, esto se debe a que, una vez establecidos los emprendimientos, el mercado ha tenido un comportamiento estable para ellos y han optado por no tomar otras decisiones. 


\section{Conclusiones}

A partir de los resultados descritos anteriormente, puede concluirse que los emprendimientos iniciados y consolidados en los últimos años en Ecuador, cuentan mayormente con líderes cuyo perfil es congruente con la gerencia Integral, al poder varios de los rasgos que distinguen este tipo de liderazgo. Ello trae como consecuencia le creciente auge de estos emprendimientos y su proliferación en casi todo el país, teniendo como resultado una empresa exitosa, indistintamente del ramo en que se desempeña.

Esto nos deja ver cómo el uso de la gerencia integral y sus planteamientos, crea líderes emprendedores exitosos, cuyas iniciativas promueven vigorosamente el mercado interno en el país.

\section{Recomendaciones}

Es válido recomendar la implementación de los principios de la Gerencia Integral dentro de las empresas públicas y privadas, indistintamente de su tamaño y del ramo al cual se dediquen. Esto en vista de que toda organización requiere de líderes que puedan gerenciar de forma adecuada los recursos y actividades de la empresa, a los fines de obtener mayores ganancias y posicionarse cada vez mejor ante la competencia.

Al margen de los planteamientos anteriores, que se observa una muy escasa presencia de emprendimientos productivos o de manufactura de cualquier rubro. Esto llama la atención en la medida en la que implica un crecimiento del sector comercial (de bienes y servicios) sin que se evidencie una base productiva que sustente dicha actividad comercial. Esto podría no ser beneficioso para las situaciones económicas del país a mediano y largo plazo, por ende, se recomienda profundizar en estudios que aborden esta temática, y que puedan generar propuestas que aporten al sector productivo.

\section{Referencias bibliográficas}

Araque, W. (2015). Emprendimiento en Ecuador. Obtenido de www.ekosnegocios.com: http://www.ekosnegocios.com/revista/pdfTemas/1133.pdf 
Bernal, C. (2010). Metodología de la investigación: Administración, economía, humanidades y ciencias sociales (3a ed.). Bogotá: Pearson Educación / Prentice Hall.

García, G., Bolívar, J., \& Roa, R. (2011). Gerencia integral para el siglo XXI: Prácticas para mejorar la competitividad de las organizaciones. Bogotá: Universidad EAN.

Hernández, R., Fernández, C., \& Baptista, P. (2010). Metodología de la Investigación (5a ed.). México D.F.: Mc Graw Hill /Interamericana Editores, S.A. de C.V.

Lederman, D., Julián, M., Pienknagura, S., \& Rigolini, J. (2014). El emprendimiento en América Latina: Muchas empresas y poca innovación. Washington D.C.: Banco Internacional de Reconstrucción y Fomento / Banco Mundial.

Monje, C. (2011). Metodología de la investigación cuantitativa y cualitativa: Guía didáctica. Neiva: Universidad Surcolombiana.

Moya, P., \& Santana, S. (2016). Sobre el concepto de emprendimiento. Santiago de Chile: Laboratorio de Innovación y Emprendimiento.

Román, O., Arbeláez, G., \& Patiño, C. (2012). Gerencia integral desde la perspectiva de un modelo de planeación estratégica. Gestión \& Desarrollo, 51-78.

Sallenave, J. (2002). La gerencia integral: No le tema a la competencia, témale a la incompetencia. Bogotá: Norma. 